\title{
Antifungal Properties of Copper Nanoparticles against Aspergillus niger
}

\author{
${ }^{1}$ Department of Biochemistry, University of Agriculture, Faisalabad, Pakistan \\ ${ }^{2}$ Department of Chemistry, Government College University, Faisalabad, Pakistan \\ ${ }^{3}$ Department of Bioinformatics and Biotechnology, Government College University, Faisalabad, Pakistan \\ ${ }^{4}$ Department of Botany, Government College University, Faisalabad, Pakistan \\ ${ }^{5}$ Department of Computer Science, University of Gujrat, Pakistan
}

Salman Maqsood ${ }^{1 *}$, Saba Qadir ${ }^{2}$, Asim Hussain ${ }^{1}$, Asma Asghar ${ }^{1}$, Rubab Saleem ${ }^{3}$, Sara Zaheer ${ }^{4}$, Noman Nayyar

\begin{abstract}
DOI: $\underline{10.36348 / \text { sijb.2020.v03i04.002 }}$
| Received: 02.04.2020 | Accepted: 09.04.2020 | Published: 14.04.2020
\end{abstract}

*Corresponding author: Salman Maqsood

\section{Abstract}

Except in severe conditions, A. niger it is a highly resistant fungal strain and causes many health issues with respect to asthma and allergy in humans. The fungus, known as the black mold in fruit and vegetables, also leads to the degradation of foodstuffs. Nanomaterials like nanoparticles of different metals are in progress against many diseases and microbial strains. Copper nanoparticles are one of them used against different microbial strains. The current study was designed for the production and characterization of copper nanoparticles and to evaluate their antifungal activities against $A$. niger. The production of $\mathrm{Cu}$ NPs was done by reduction method explained by Kathad and Gajera (2014) and their characterization was done by using UV-visible spectrophotometer. To evaluate their antifungal activity A. niger was grown on PDA medium with different concentrations of $\mathrm{Cu}$ NPs. The growth rate of $A$. niger was evaluated at different time intervals and compared with control. The UV-visible spectra indicated the presences of Cu NPs as maximum absorbance peak was observed at $590 \mathrm{~nm}$. A significant inhibitory effect was observed on growth rate of A. niger at different concentrations of $\mathrm{Cu}$ NPs. Comparative analysis of these concentrations depicted that maximum inhibition was observed at $1.5 \%$ concentration of CU NPs. It is concluded that copper nanoparticles show antifungal activity and their activity is concentration dependant.

Keywords: Nanoparticles, Antifungal, Copper, A. niger, Characterization.

Copyright @ 2020: This is an open-access article distributed under the terms of the Creative Commons Attribution license which permits unrestricted use, distribution, and reproduction in any medium for non-commercial use (NonCommercial, or CC-BY-NC) provided the original author and source are credited.

\section{INTRODUCTION}

The spoilage of crops and breeding goods causes significant losses on food and beverage sectors in the global annual revenue. Mold spoilage can also be a food safety problem as such molds contain mycotoxins or allergens. Fungal spoilage classes include xerophilic fungi, heat-resistant fungi, condomprotective fungi, and psychrophilic fungi [1]. Recent epidemiological studies indicate that pathogenic fungal microorganisms like the Aspergillus genus have dramatically increased fungicide resistance. The fungus may be absorbed in the skin, swallowed or inhaled by animals, and cause aspergillosis in humans [2]. Aspergillus niger is a fungal strain which can cause allergies and asthma, particularly when exposed to indoors for longer periods of time. This fungus, called black mold, is mostly found in fruits and vegetables. This fungus also occurs on the painted surfaces of buildings that degrade, flatten and disperse the coating [3]. This is an extremely resistant fungus which grows in a variety of conditions, $\mathrm{pHs}$, salt levels and humidity [4].

Owing to widespread use of antifungal products against fungal infections, fungi have resistance to most antifungal products that are commercially available and therefore make infectious diseases difficult to treat. The widespread and systematic use of antifungal products against spreadable pathogens has increased the fungal resistance issue and is therefore a major concern for treatment of these infections [5]. For this question, Nanoparticles have, because of their particular sizes and properties from the last few decades, been synthesized, engineered and used on a commercial level as a remedy for fungal diseases [6].

In terms of their distribution compared with the remaining bulk materials, nanoparticles have a distinctive characteristic [7]. Nanoparticles are entirely special in material science or are of value-added materials, such as their shape, scale, delivery and distribution etc. Such nanoparticles are currently 
commonly used in different areas [8]. Based on factors such as special physical, optical and chemical properties, nanoparticles belong to modern mechanization. These are effective as antibacterial, anti-viral and antifungal agents for various applications in the fields of catalyst, photonics, electronics and medicine [9].

Due to its possible applications in the development of innovative abilities [10], the processing of nanoparticles is a vast area of study. All of them contains copper nanoparticles. In current materialistic industry, copper nanoparticles have major effects. Copper nanoparticles are non-toxic to humans and highly selective against microorganisms at low concentrations [11]. The nuclear stage offers new opportunities and resources for disease treatment [12]. These particles are typically used as pesticides, fungicides, antimicrobials and medicine and biomedical applications $[13,14]$. These particles are often used for the use of pharmacological items. They have been shown to be antifungal against Aspergillus niger and are reliant on fungal diseases [15]. Copper appears to encourage resistance to fungi in contrast to the other agents [16].

Multiple methods may be applied to prepare copper nanoparticles. Another of them is the reduction method. The method of reduction can be applied to solve the toxicity issues and has the potential to increase material quality [17]. Synthesis of copper nanoparticles can be induced by Brassica juncea, Medicago sativa, Helianthus annus and Tridax procumbens.

The present study was conducted for the characterization of Copper nanoparticles and to investigate their antifungal properties against $A$. niger.

\section{MATERIALS AND METHODS}

Sterilized glass wares, analytic grade and pure chemical substance were used in this experiment for the synthesis of copper nanoparticles and media preparation for growth of A. niger. Copper Sulphate, Cetyltrimethylammonium bromide, Deionized water, Ascorbic acid, D-Glucose, Chloramphenicol (Merck, Germany), Potato dextrose agar (PDA) and Agar Agar (Sigma-Aldrich, UK) were prepared by following their protocols.

\section{Preparation of CuNPs}

Copper nanoparticles were prepared by adopting reduction method explained by Kathad and Gajera [18]. 0.01 M of $\mathrm{CuSO}_{4} \cdot 5 \mathrm{H}_{2} \mathrm{O}$ and $0.11 \mathrm{M}$ of ascorbic acid were dissolved in $100 \mathrm{~mL}$ of deionized water in a $250 \mathrm{~mL}$ flat-bottom flask equipped with a hot plate, a small magnetic stir bar, and a thermometer. The $0.03 \mathrm{M}$ of CTAB was introduced into the solution with rapid stirring at room temperature. Aqueous $\mathrm{NaOH}$ was employed to control $\mathrm{pH}$ 6.5. The mixture was agitated at $85{ }^{\circ} \mathrm{C}$ without any inert gas protection. The temperature was monitored with thermometers. Copper particles were separated by filtrating, washed with deionized water and ethanol.

\section{Characterization of $\mathrm{Cu}$ NPs}

Characterization of $\mathrm{Cu}$ NPs was done by UVVisible spectrophotometer analysis [19]. First of all, cuvettes were taken out, cleaned properly and then filled with deionized water. These cuvettes were then placed carefully and accurately for spectrophotometric analysis and value observed was nullified. After the addition of copper sulphate solution and ascorbic acid immediately $1 \mathrm{~mL}$ of deionized water was mixed with $2 \mathrm{~mL}$ of nano solution in spectrophotometer. Five samples were taken at duration of 15 minutes, 30 minutes, 45 minutes, 60 minutes and 24 hours. Scanning was done to observe the absorption peak at different wavelengths from 290-690nm separately for each sample [20].

\section{Media preparation for $A$. niger}

Potato dextrose agar (PDA) medium was used as a growth media for A. niger. PDA media was prepared by adding $4 \mathrm{~g}$ PDA and $2 \mathrm{~g}$ agar-agar in a beaker and dissolved in distilled water by heating. $\mathrm{pH}$ of medium was adjusted at 6 and volume up to 1000 $\mathrm{mL}$. Medium was poured on Petri plates and sterilized by autoclave for 2 hours [21].

\section{Growth of $A$. niger}

A. niger culture was taken from the preserved slants streaked on the plates that containing the PDA. After streaking, the plates were kept in incubator at $30^{\circ} \mathrm{C}$ for 72 hours. For treated groups $\mathrm{Cu}$ NPs with different concentrations $(0.5,1.0$ and $1.5 \%)$ were introduced along with $A$. niger.

\section{RESULTS AND DISCUSSION}

The present study was conducted to characterize the copper nanoparticles prepared by reduction method and to investigate their antifungal activity on A. niger. A. niger is resistant strain of the family Aspergillus and contributes to fruit and vegetables ' decay by black mold disease and also causes human health problems such as asthma and allergies [4].

\section{Characterization of Cu NPs}

Copper nanoparticles were prepared by using reduction method explained by Kathad and Gajera [18] and characterized by UV-visible spectrophotometer. Figure 1 is indicating the absorbance peak at different wavelengths $(290,390,490,590$ and $690 \mathrm{~nm})$ and different time periods (15min, 30min, 45min, 60min and $24 \mathrm{hrs}$ ). Maximum absorbance was observed at 590 $\mathrm{nm}$ at each time interval and than a decrease in absorbance was observed at $690 \mathrm{~nm}$. Maximum absorbance at $590 \mathrm{~nm}$ indicated the presence of copper nanoparticles. Similar results were observed by Usman 
et al. [22]. The progress of the CuNPs from its height at $560 \mathrm{~nm}$, which is the surface resonance band CuNPs, has been confirmed. Plasmon surface peaks from 590 to
$550 \mathrm{~nm}$ were typically seen by nanosized CuNPs. When scale increased, the most extreme peak was found to decline gradually [22].

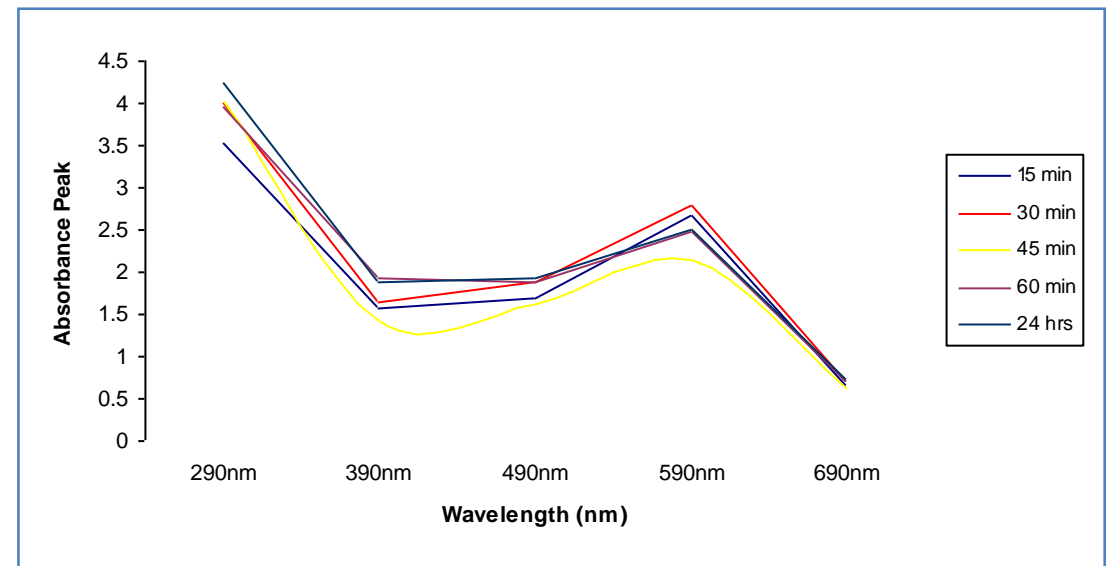

Fig-1: A line graph plotted against wavelength vs absorption peaks obtained by scanning the newly synthesized nanoparticles at different duration of time in UV-vis spectrophotometer. Analysis of CuNP's showed that the maximum absorption was observed at 590nm suggesting reduction of $\mathrm{CuSO}_{4} .5 \mathrm{H}_{2} \mathrm{O}$ into CuNP's

\section{Effect of Cu NPs on growth rate of $A$. niger}

The antifungal activity of prepared and characterized nanoparticles was observed by comparing growth rates of $A$. niger grown with different concentrations $(0.5,1$ and $1.5 \%)$ of $\mathrm{Cu}$ nanoparticles with control group. Figure 2(a) is illustrating the growth rate of $A$. niger at $0.5 \%$ concentration of $\mathrm{Cu}$ NPs. Result indicated a significant inhibition in growth of $A$. niger with respect to control $(\mathrm{p}<0.001)$.

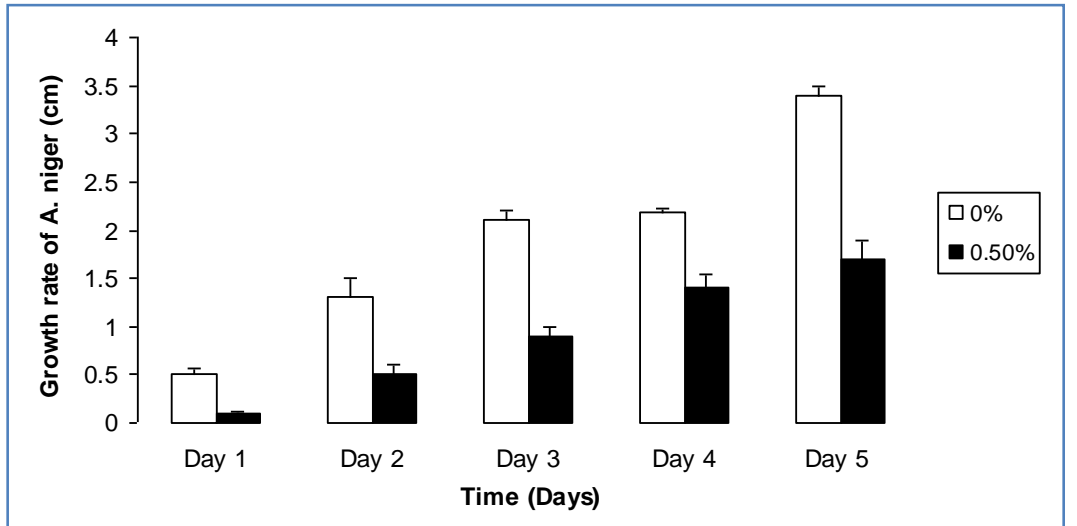

Fig-2(a): Growth rate spectrum of $A$. niger at $0.5 \%$ concentration of CuNPs. Treated groups (Black bars) indicating significant inhibitory effect of CuNPs $(0.5 \%)$ with respect to control (white bar) $(\mathbf{P}<0.001)$ at different time intervals

Figure 2(b) is indicating a highly significant inhibitory effect on growth rate of $A$. niger at $1 \%$ concentration of $\mathrm{Cu}$ NPs with respect to control.

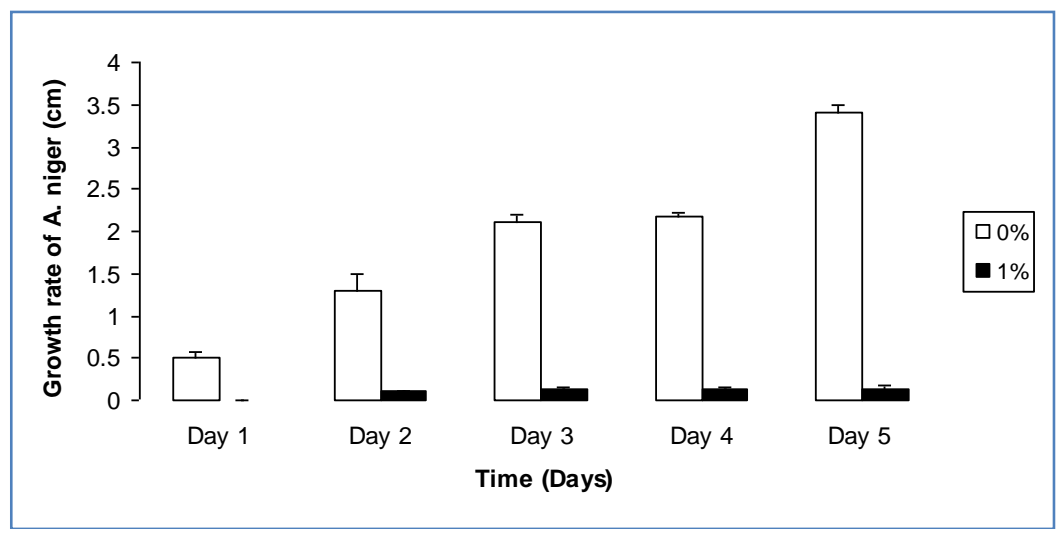


Fig-2(b): Growth rate spectrum of $A$. niger at 1.0\% concentration of CuNPs. Treated groups (Black bars) indicating significant inhibitory effect of CuNPs $(\mathbf{1 . 0 \%})$ with respect to control (white bar) $(\mathbf{P}<0.001)$ at different time intervals

Likewise figure $2(\mathrm{c})$ is indicating the growth rate of $A$. niger at $1.5 \%$ concentration of copper nanoparticles and showed highly significant inhibitory effect on growth rate of $A$. niger at $1.5 \%$ concentration.
Tantubay et al. [23] have recently reported significant anti-fungal activities in the chemical reduction of carboxymeted chitosan-stabilized copper nanoparticles in vitro against Candida tropicalis and Escherichia coli.

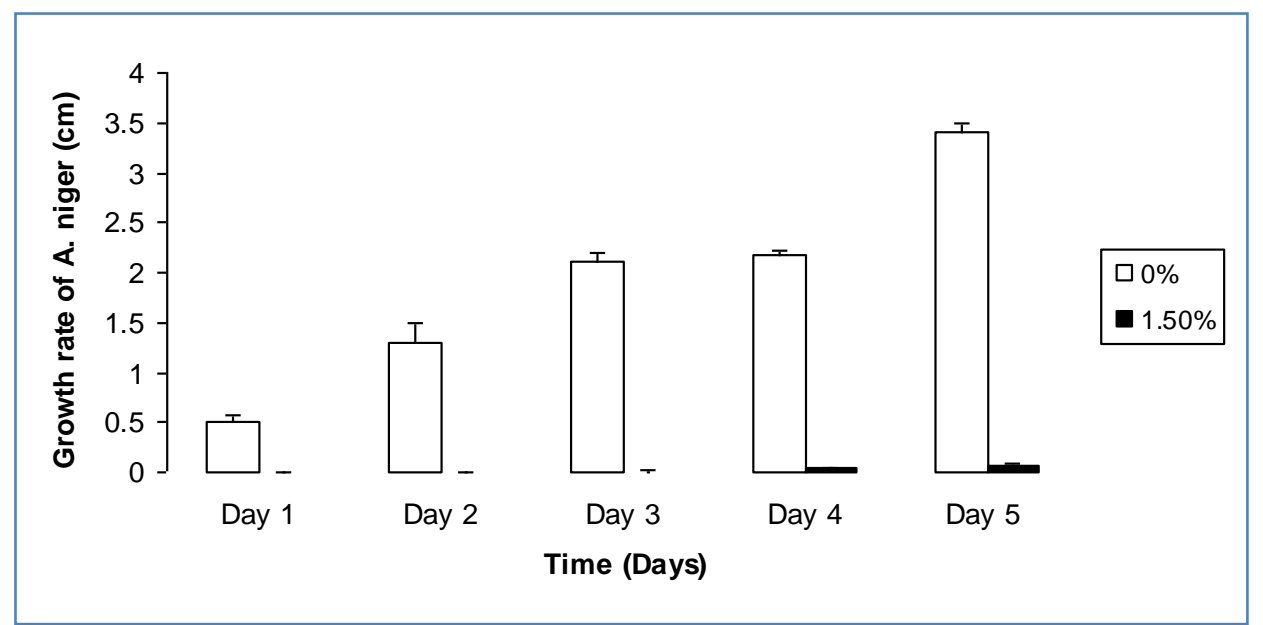

Fig-2(c): Growth rate spectrum of $A$. niger at $1.5 \%$ concentration of CuNPs. Treated groups (Black bars) indicating significant inhibitory effect of CuNPs $(1.5 \%)$ with respect to control (white bar) $(\mathbf{P}<0.001)$ at different time intervals

Figure 3 is indicating the comparative inhibitory effect of different concentrations $(0.5,1$ and $1.5 \%)$ of $\mathrm{Cu}$ NPs on growth rate of $A$. niger. Results depicted maximum inhibition at $1.5 \%$ concentration of
$\mathrm{Cu}$ NPs which also indicate that antifungal activity of $\mathrm{Cu}$ NPs is dose/concentration dependant as mentioned earlier [15].

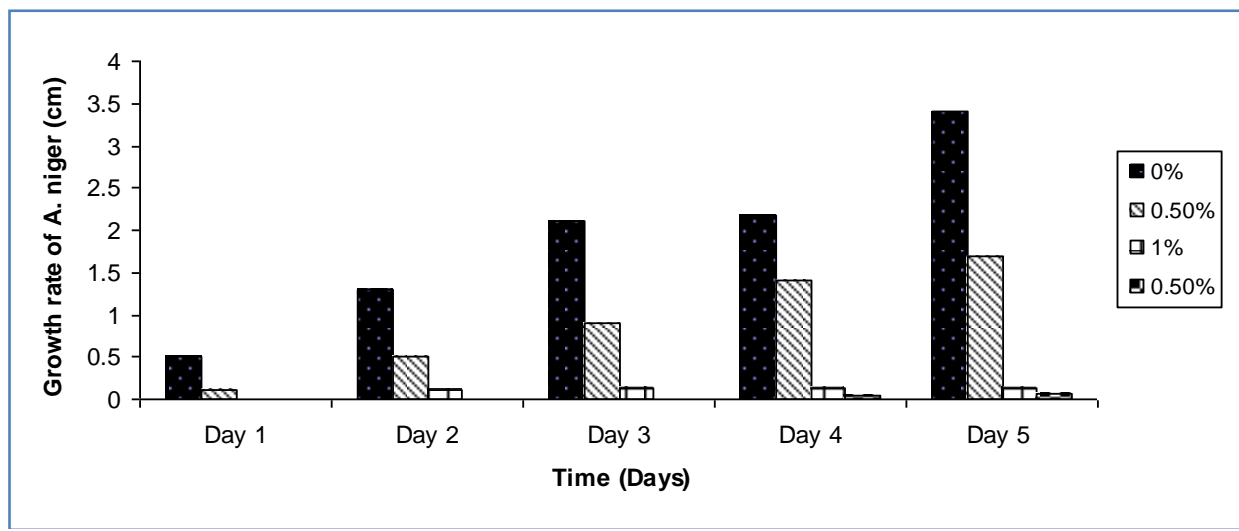

Fig-3: Growth rate spectrum of A. niger for comparative analysis at different concentrations $(0.5,1.0$ and $1.5 \%)$ of CuNPs with maximum inhibition at $0.5 \%$ concentration

\section{CONCLUSIONS}

The use of nanoparticles is common in practice now days. Due to their small size and large surface area nanoparticles are used for therapeutically and diagnostic purposes. Copper nanoparticles are one of them. They act as antibiotic, anti-microbial and antifungal agents. In our research work antifungal activity of $\mathrm{Cu}-\mathrm{NPs}$ was observed against $A$. niger and it was concluded that copper nanoparticles showed antifungal activity against $A$. niger and this activity is concentration dependant.

\section{Author's Note}

Salman Maqsood and Asim Hussain contributed equally and thus shares first authorship.

\section{REFERENCES}

1. Rico-Munoz, E., Samson, R. A., \& Houbraken, J. (2019). Mould spoilage of foods and beverages: Using the right methodology. Food microbiology, 81, 51-62.

2. Person, A. K., Chudgar, S. M., Norton, B. L., Tong, B. C., \& Stout, J. E. (2010). Aspergillus niger: an unusual cause of invasive pulmonary aspergillosis. Journal of Medical Microbiology, 59(Pt 7), 834.

3. Gaylarde, C. C., Morton, L. H. G., Loh, K., \& Shirakawa, M. A. (2011). Biodeterioration of external architectural paint films-A 
review. International Biodeterioration \& Biodegradation, 65(8), 1189-1198.

4. Velazquez-Herrera, F. D., Fetter, G., Rosato, V., Pereyra, A. M., \& Basaldella, E. I. (2018). Effect of structure, morphology and chemical composition of $\mathrm{Zn}-\mathrm{Al}, \mathrm{Mg} / \mathrm{Zn}-\mathrm{Al}$ and $\mathrm{Cu} / \mathrm{Zn}-\mathrm{Al}$ hydrotalcites on their antifungal activity against A. niger. Journal of Environmental Chemical Engineering, 6(2), 33763383.

5. Rubina, M. S., Vasil'kov, A. Y., Naumkin, A. V., Shtykova, E. V., Abramchuk, S. S., Alghuthaymi, M. A., \& Abd-Elsalam, K. A. (2017). Synthesis and characterization of chitosan-copper nanocomposites and their fungicidal activity against two sclerotia-forming plant pathogenic fungi. Journal of Nanostructure in Chemistry, 7(3), 249-258.

6. Shankar, S. S., Ahmad, A., \& Sastry, M. (2003). Geranium leaf assisted biosynthesis of silver nanoparticles. Biotechnology progress, 19(6), 1627-1631.

7. Banerjee, P., Satapathy, M., Mukhopahayay, A., \& Das, P. (2014). Leaf extract mediated green synthesis of silver nanoparticles from widely available Indian plants: synthesis, characterization, antimicrobial property and toxicity analysis. Bioresources and Bioprocessing, 1(1), 3.

8. Kaviya, S., Santhanalakshmi, J., \& Viswanathan, B. (2011). Green synthesis of silver nanoparticles using Polyalthia longifolia leaf extract along with D-sorbitol: study of antibacterial activity. Journal of nanotechnology, 2011.

9. Janardhanan, R., Karuppaiah, M., Hebalkar, N., \& Rao, T. N. (2009). Synthesis and surface chemistry of nano silver particles. Polyhedron, 28(12), 25222530.

10. Panigrahi, T. (2013). Synthesis and characterization of silver nanoparticles using leaf extract of Azadirachta indica (Doctoral dissertation).

11. Savithramma, N., Rao, M. L., Rukmini, K., \& Devi, P. S. (2011). Antimicrobial activity of silver nanoparticles synthesized by using medicinal plants. International Journal of ChemTech Research, 3(3), 1394-1402.

12. Kanhed, P., Birla, S., Gaikwad, S., Gade, A., Seabra, A. B., Rubilar, O., \& Rai, M. (2014). In vitro antifungal efficacy of copper nanoparticles against selected crop pathogenic fungi. Materials Letters, 115, 13-17.

13. Chakraborty, P., Adhikary, J., Chatterjee, S., Biswas, B., \& Chattopadhyay, T. (2016). Facile synthesis of copper sulfide nanoparticles: antibacterial and antifungal activity study. Rasayan J. Chem, 9(1), 77-83.
14. Ravichandran, R. (2009). Nanotechnology-based drug delivery systems. Nano Biotechnology, 5(1-4), 17-33.

15. Johari, S. A., Kalbassi, M. R., \& Yu, I. J. (2014). Inhibitory effects of silver zeolite on in vitro growth of fish egg pathogen, Saprolegnia sp. Journal of Coastal Life Medicine, 2(5), 357361.

16. Xia, Z. K., Ma, Q. H., Li, S. Y., Zhang, D. Q., Cong, L., Tian, Y. L., \& Yang, R. Y. (2016). The antifungal effect of silver nanoparticles on Trichosporon asahii. Journal of Microbiology, Immunology and Infection, 49(2), 182-188.

17. Gültekin, D. D., Güngör, A. A., Önem, H., Babagil, A., \& Nadaroğlu, H. (2016). Synthesis of copper nanoparticles using a different method: determination of its antioxidant and antimicrobial activity. Journal of the Turkish Chemical Society, Section A: Chemistry, 3(3), 623-636.

18. Kathad, U., \& Gajera, H. P. (2014). Synthesis of copper nanoparticles by two different methods and size comparison. Int J Pharm Bio Sci, 5(3), 533540 .

19. Kailasa, S. K., Koduru, J. R., Desai, M. L., Park, T. J., Singhal, R. K., \& Basu, H. (2018). Recent progress on surface chemistry of plasmonic metal nanoparticles for colorimetric assay of drugs in pharmaceutical and biological samples. $\operatorname{Tr} A C$ Trends in Analytical Chemistry, 105, 106-120.

20. Parveen, S., Wani, A. H., Shah, M. A., Devi, H. S., Bhat, M. Y., \& Koka, J. A. (2018). Preparation, characterization and antifungal activity of iron oxide nanoparticles. Microbial pathogenesis, 115, 287-292.

21. Prado, M. R., Brito, É. H., Brilhante, R. S., Cordeiro, R. A., Leite, J. J., Sidrim, J. J., \& Rocha, M. F. (2008). Subculture on potato dextrose agar as a complement to the broth microdilution assay for Malassezia pachydermatis. Journal of microbiological methods, 75(2), 341-343.

22. Usman, M., Ahmed, A., Yu, B., Peng, Q., Shen, Y., \& Cong, H. (2019). Photocatalytic potential of bioengineered copper nanoparticles synthesized from Ficus carica extract for the degradation of toxic organic dye from waste water: Growth mechanism and study of parameter affecting the degradation performance. Materials Research Bulletin, 120, 110583.

23. Tantubay, S., Mukhopadhyay, S. K., Kalita, H., Konar, S., Dey, S., Pathak, A., \& Pramanik, P. (2015). Carboxymethylated chitosan-stabilized copper nanoparticles: a promise to contribute a potent antifungal and antibacterial agent. Journal of Nanoparticle Research, 17(6), 243. 